\title{
Synthesis and Characterization of Dose-Dependent Antioxidants and Antimicrobial Activity of Phloretin Loaded PLGA Nanoparticles
}

\author{
Thangavelu Ranjanamala $^{1}$ (D), KrishananVanmathiselvi ${ }^{1 *}{ }^{*}$, , Sangeetha C Casimeer ${ }^{2}$ (D), Alaa Yousef \\ Ghidan 3 iD
}

1 PG and Research department of microbiology, Sri Akilandeswari Women's College, Wandiwash-604408, Tamil Nadu, India; ranjanamalasankar@gmail.com (T.R.); kvmsel@gmail.com (K.V.);

2 Department of Physics, Sri Padmavati Mahila Visvavidyalayam University, Tirupati, Andhra Pradesh 517502, India; carosangee@gmail.com (C.C.S.);

3 Nanotechnology and Microbiology, Research and Development Center, The Higher Council for Science and Technology, Amman, 11941, Jordan; alaayghidan@gmail.com (A.Y.G.);

* Correspondence: kvmsel@gmail.com;

Received: 12.05.2021; Revised: 20.06.2021; Accepted: 25.06.2021; Published: 8.08.2021

Abstract: This aim of the study combines and differentiates Phloretin-encapsulated PLGA (poly (dllactide-coglycolide) nanoparticles and assesses their antioxidant and antibacterial properties. Phloretin is a possible active ingredient with a wide range of therapeutic effects. The process of emulsification was used to make nanoparticles. Physicochemical properties were measured by Particle size, polydispersity index, and zeta-potential investigated by dynamic light scattering (DLS), X-ray diffraction, FTIR, TEM, and AFM. FTIR results proven that phloretin formulated PLGA NPs exhibited functional groups. The Zeta potential size analyzer results of the optimal NPs size were $98.7 \mathrm{~nm}$. The crystallinity nature peaks related to Phloretin-PLGA NPs were confirmed by XRD. Antioxidant properties of free and encapsulated nanoparticles were determined by DP, method. Relatively small polymeric nanoparticles were obtained containing phloretin. DPPH radical scavenging activity of the nanoparticles was found to be $71 \%$ at a $1 \mathrm{mg} / \mathrm{ml}$ concentration. It also had antibacterial properties when examined using the good diffusion approach against Negative species. In conclusion, our findings show that encapsulated PLGA phloretin nanoparticles with continuous release properties retain antioxidant and antimicrobial activity, implying therapeutic potential.

Keywords: Phloretin; PLGA; nanoformulation; antioxidants; antibacterial activity.

(C) 2021 by the authors. This article is an open-access article distributed under the terms and conditions of the Creative Commons Attribution (CC BY) license (https://creativecommons.org/licenses/by/4.0/).

\section{Introduction}

Nanotechnology's use in medicine provides a vital method for disease diagnosis and treatment. A variety of submicron materials have been developed and engineered specifically to combat cancer. Its applications pushed the development of new therapeutics, drug delivery vehicles, and theranostics agents. Renewable and biocompatible polymers based on natural and/or synthetic materials have been used to make nanoparticles (NPs) for drug delivery applications. In comparison to natural materials, synthetic polymers can be manufactured with high purity in an exact and well-controlled production process.[1]

PLGA (poly(lactic-co-glycolic acid)) is a synthetic copolymer approved by the FDA for the use of medicinal and pharmaceutical industries, including drug delivery [2]. Biodegradable polymers are widely used in the field of drug delivery in the midst of current nanomaterials. Because of its easy hydrolyzing property inside the body, PLGA (Poly Lacto 
Co-glycolic acid) is one of the best-featured biopolymers frequently used for drug delivery.[3] Biocompatible and biodegradable, PLGA is a material that can be used in a variety of applications. When hydrolysed, it converts into non-toxic oligomeric and monomeric lactic and glycolic acids and then excreted as carbon dioxide and water [4,5]. The degradation rate of this copolymer can be altered by adjusting the molar ratios of lactic and glycolic acids in the polymer chain. [6]. PLGA nanoparticles may help drugs cross biological barriers, including the blood-brain barrier, the gastrointestinal mucosa, the nasal mucosa, and ocular tissue[7]. As a result, this copolymer has been meticulously used as a Nano component. The hydrophobic polyphenolic compound phloretin(3-(4-hydroxyphenyl)-1-(2,4,6 trihydroxy phenyl) propan-1one (MF: C15H14O5; MW $274.3 \mathrm{~g} / \mathrm{mol}$ ) [8,9]. Phloretin belongs to the chalcone class of flavonoids and is found in nature. Since phloretin is abundant in apples and strawberries, it has become a staple of human nutrition. Flavonoids are phenolic-structured natural bioactive components found in a variety of plant forms [10]. Recently, some scholars have improved the absorption of phloretin in vivo by preparing different dosage forms (such as self-nanoemulsion [11], liposome, and microemulsion [12]) according to the low bioavailability of phloretin. It is typically found in the apple peel (80-420 mg/kg) and pulp (16-20 mg/kg); however, the concentration varies greatly depending on the apple variety [13]. Phloretin has been shown to have anti-inflammatory, anti-oxidative, and anti-cancer properties in previous studies $[14,15,16]$. According to recent reports, phloretin can be converted to phlorizin, trilobactin, phloretin 20-O-xyloglucoside, sieboldin, 3hydroxyphlorizin, and 3-hydroxyl phloretin. [17].

\section{Materials and Methods}

\subsection{Chemicals and nanoformulation.}

Phloretin was procured from Cayman Chemical Company (Ann Arbor, USA). Polylactic acid and polyglycolic acid are two different types of acid. PLGA is an abbreviation for polylactic acid (MW: 5000- 15,000), $\alpha, \alpha$-diphenyl- $\beta$-picrylhydrazyl (DPPH), 2,2'azinobis-(3-ethylbenzothiazoline-6-sulfonic acid) (ABTS), Sodium acetate, sodium carbonate, butanol, sodium hydroxide, acetone, ethanol, chloroform, and aluminum chloride were obtained from Merck, Mumbai, India. Mannitol salt, Mueller Hinton Agar were procured from Hi-media Ltd. All the solvents were used for HPLC. Nanoparticles were synthesized using Milli-Q grade water.

\subsection{Phloretin-Loaded PLGA Nanoparticles Fabrication (Phl-PLGA NPS).}

With slight adjustments, Phl-PLGA NPs are fabricated using oil in water single emulsion solvent evaporation technique [18]. $50 \mathrm{mg}$ of PLGA is dissolved in $5 \mathrm{ml}$ of dichloromethane and acetone (dichloromethane/acetone 3/2) to make a well-incorporated PLGA solution $(10 \mathrm{mg} / \mathrm{ml})$ with the PLGA completely dissolved. $50 \mathrm{mg}$ of phloretin was merged with the PLGA solution and sonicated at $200 \mathrm{~W}$ for 2 min (organic phase) to develop a primary emulsion. The primary emulsion was then slowly injected into the BSA solution (1 percent w/v) (aqueous phase), assisted by 4 minutes of sonication at $200 \mathrm{~W}$. It will be time to make the final oil/water $(\mathrm{O} / \mathrm{W})$ emulsion. $15 \mathrm{~mL}$ deionized water was used to disperse the final $\mathrm{O} / \mathrm{W}$ emulsion. Nanoparticles were obtained after centrifugation at 14,000 rpm for 30 minutes, and the supernatant was removed. The extracted NPs were then centrifuged three times at $10,000 \mathrm{rpm}$ for 20 minutes to remove the deionized water. Finally, the nanoparticles were resuspended in deionized water and vacuum frozen, with the freeze-dried nanoparticles being 
deposited at $4{ }^{\circ} \mathrm{C}$. The physicochemical properties of phloretin-loaded PLGA nanoparticles (PHL-PLGA-NPs) were confirmed by Transmission Electron Microscopy (TEM), dynamic light scattering (DLS), Fourier Transform Infrared Spectroscopy (FTIR), XRD, and NMR analysis [19].

\subsection{Physicochemical analysis.}

\subsubsection{XRD analysis.}

The phase purity and crystallinity of the synthesized Phl-PLGA NPs were determined using XRD. The mixed solution of synthesized Phl-PLGA NPs was syringe filtered, dried, and analyzed using an X'pert PRO X-ray diffractometer (Netherlands) with a $\mathrm{Cu}$ k1 radiation source and a voltage of $40 \mathrm{kV}$ and an electric current of $20 \mathrm{~mA} .45 \mathrm{kV}, 9 \mathrm{~mA}$, and a voltage of $2 \theta$.

\subsubsection{FT-IR spectroscopy.}

Using Fourier transform infrared spectroscopy, the KBR method was used to detect the functional group present in the synthesized nanoparticles (FTIR PerkinElmer, USA). The nanoparticles were homogeneously mixed with $\mathrm{KBr}$ and compressed into discs, which were traced using an FT-IR spectrophotometer in the 400-4000 cm1 range.

\subsubsection{Dynamic light scattering measurements (DLS).}

The measurements of DLS were used to utilize the particle size and charge of the synthesized Phl-PLGA NPs. The synthesized sample was filtered using a syringe filter with a $0.20 \mathrm{~m}$ range, and the particle size was measured at a $90^{\circ}$ scattering angle and analyzed at 25.2 ${ }^{\circ} \mathrm{C}$ (Horiba-DLS-7100E, Japan). For the analysis, a count rate of $162 \mathrm{kcps}$ was used. Prior to analysis, the samples were diluted with Milli Q water. The intensity of scattered light was used to present the results. For the zeta-potential study, the electrode voltage was set to $3.3 \mathrm{~V}$ with a $0.528 \mathrm{mS} / \mathrm{cm}$ conductivity range. Readings were taken with the Horiba SZ-100 for Windows [Z Type] Ver2.00 software.

\subsubsection{Scanning electron microscopy (SEM).}

The shape and surface characteristics of phloretin, as well as PHL-PLGA NPs, were recorded using SEM (Hitachi, Japan). Double-sided adhesive carbon tapes were used to mount samples on metal stubs. Samples were coated with a gold coating and viewed under a $30 \mathrm{kV}$ SEM.

\subsubsection{Transmission electron microscopy (TEM)}

The morphology of phloretin and PHL-PLGA NPs nanoparticles was investigated using a transmission electron microscope set to $160 \mathrm{Kv}$ (FEI-TECNAI G2-20 TWIN, USA). Using the double deionized water, the lyophilized nanoparticles were revitalized and that had been correctly diluted and sonicated for 2 minutes. One drop was placed on a Formvar-coated copper grid, and the excess was removed with filter paper. The image was captured and analyzed using imaging viewer software after it had completely dried at room temperature. 
2.4. Antioxidant activity of Phl-PLGA NPs.

\subsubsection{DPPH radical scavenging activity.}

DPPH radical scavenging activity free radical scavenging activity of Phloretin and PhlPLGA NPs nanoparticles was determined according to the method using DPPH [20]. The ABTS (2,2-Azino-bis (3-ethylbenzthiazoline-6-sulfonic acid radical) and DPPH (1,1diphenyl-2-picrylhydrazyl radical) assays are the most widely used and rapid methods for estimating antioxidant activity.[21] In brief, DPPH (1, 1-diphenyl-2-picrylhydrazyl) (0.004 percent DPPH) solution $(0.2 \mathrm{mM}$ in methanol)- $3 \mathrm{~mL}$ was mixed with different concentrations of nanoparticles $(0.1-1 \mathrm{mg} / \mathrm{ml})$. The mixture was vortexed and kept at room temperature for 45 mins. Each nanoparticle component's proton contributes activity was measured at $517 \mathrm{~nm}$, resulting in a decrease in solution absorbance. For greater free radical scavenging activity, the reaction mixture should be less repulsive. The positive control was vitamin $\mathrm{C}$ (L-ascorbic acid). The radical scavenging activity of DPPH was calculated using the formula:

\section{Antioxidant activity $(\%)=\left(\mathrm{A}_{0}-\mathrm{A}_{1}\right) \mathrm{A}_{0} \mathrm{x} 100$}

Wher $\mathrm{A}_{0}$ is the control pressure, and $\mathrm{A}_{1}$ is the pressure of extract or standard sample.

\subsubsection{ABTS radical scavenging activity.}

With the reference of Re et al., ABTS free radical scavenging capacity is carried out with some modifications [22]. ABTS in water and $2.45 \mathrm{mmol} / \mathrm{L} \mathrm{K}_{2} \mathrm{~S}_{2} \mathrm{O}_{8}$ was liquefied to a concentration of $7 \mathrm{mM}$. Before using, the mixture is kept in the dark for 12-16 hours at room temperature. Methanol was used to dilute the ABTS solution until the absorbance at $734 \mathrm{~nm}$ was 0.700 .02 . For 6 minutes, $5 \mathrm{~mL}$ of diluted ABTS was added to $0.5 \mathrm{~mL}$ of diluted samples. The samples were assessed by comparing them to control (containing $5 \mathrm{~mL}$ of ABTS solution and $0.5 \mathrm{~mL}$ of ethanol). A BTS + scavenging effect $(\%)=((\mathrm{AB}-\mathrm{AA}) / \mathrm{AB}) \times 100(2)$, where, $\mathrm{AB}$ is absorbance of ABTS radical + methanol; AA is absorbance of ABTS radical + sample extract/standard. Each sample was measured in triplicate, and the average was calculated.

\subsubsection{Hydrogen peroxide scavenging assay.}

Arai et al., described the $\mathrm{H}_{2} \mathrm{O}_{2}$ radical scavenging assay was carried out in this study [23]. The test is done by combining different concentrations of Phloretin and Phl-PLGA NPs and Standard (Ascorbic acid) $\left(12.5-100 \mu \mathrm{g} / \mathrm{mL}\right.$ ) to $0.6 \mathrm{~mL}$ of $\mathrm{H}_{2} \mathrm{O}_{2}$ prepared in phosphate buffer solution modify to $(40 \mathrm{mM}, 7.4 \mathrm{pH}) . \mathrm{H}_{2} \mathrm{O}_{2}$ alone was used as a blank. Along with phosphate buffer $(40 \mathrm{mM}, 7.4 \mathrm{pH})$ Ascorbic acid was used as a standard. The supernatant was kept incubated for $10 \mathrm{~min}$ at room temperature, and samples were vortexed. The potency of the sample was determined by UV-visible spectroscopy and read at $610 \mathrm{~nm}$. IC50 (\%) $=100 \times\left(\mathrm{AC}_{\mathrm{C}}\right.$ $\left.-A_{s}\right) / A_{c}$, where $A_{s}$ and $A_{0}$ are the values for the absorbance of the sample and absorbance of the negative control, respectively. Tests were in triplicate.

\subsubsection{Hydrogen radical scavenging assay.}

Hydroxyl radical (OH) scavenging activity was performed as Kumar et al., 2015[24] reported with some modifications. By fresh reaction mixture composed of $\mathrm{FeSO}_{4}$ and $\mathrm{H}_{2} \mathrm{O}_{2} \mathrm{OH}$ radicals were performed using a usual Fenton reaction. To the mixture $1.2 \mathrm{~mL}$ of $0.1 \mathrm{~mL}$ of phosphate buffer and the samples (Phloretin and Phl-PLGA NPs and Standard Ascorbic acid) 
at various concentrations draw up from $12.5-100 \mu \mathrm{g} / \mathrm{mL}$ were taken. $1 \mathrm{~mL}$ of $3 \%$ TCA and TBA were mixed and kept steaming for $10 \mathrm{~min}$ in a water bath after the mixture had been cooled. Absorbance was measured at $532 \mathrm{~nm}$. IC50 $(\%)=100 \times\left(\mathrm{Ac}_{\mathrm{c}}-\mathrm{A}_{\mathrm{s}}\right) / \mathrm{A}_{\mathrm{c}}$, where $\mathrm{As}_{\mathrm{s}}$ and $A_{0}$ are the values for the absorbance of the sample and absorbance of the negative control, respectively. Tests were performed in triplicate.

\subsubsection{Superoxide radical scavenging activity.}

Superoxide radical scavenging assay was performed by the method described by Pool et al. (2012) [25]. Based on NADH oxidation, the Nitro blue tetrazolium to a violet-colored complex formation due to the production of superoxide radicals by the Phenozine methosulphate system, reduction of color is due to the antioxidant scavenging factor of the sample. The mixture was containing phosphate buffer (0.1 M, pH- 8), NADH (73 $\mu \mathrm{M}, \mathrm{PMS}$ $(15 \mu \mathrm{M})$ NBT $(50 \mu \mathrm{M})$, and various concentrations of PHL, Phl-PLGA NPs and Standard (Ascorbic acid) in methanol $(12.5-100 \mu \mathrm{g} / \mathrm{mL})$. The mixture was incubated for 5 minutes at ambient conditions, and the intensity was recorded at $560 \mathrm{~nm}$. The inhibition ratio was calculated using the formula, IC50 $(\%)=100 \times\left(\mathrm{Ac}_{\mathrm{c}}-\mathrm{A}_{\mathrm{s}}\right) / \mathrm{A}_{\mathrm{c}}$, where $\mathrm{A}_{\mathrm{s}}$ and $\mathrm{A}_{0}$ are the values for the absorbance of the sample and absorbance of the negative control, respectively. Tests were performed in triplicate.

\subsection{Antibacterial property by well diffusion of Phl-PLGA.}

Phloretin's antimicrobial activity was discovered by Barreca et al. (2014) [26]. Antimicrobial activities will be evaluated using a pathogenic gram-negative drug resistance bacterial strain [27]. A modified agar-well diffusion method was used to test the antibacterial property. A nutrient broth was prepared, and the bacteria were subcultured to obtain fresh culture. $50 \mathrm{~L}$ of stock culture was mixed with $950 \mathrm{~L}$ of sterile Muller Hinton broth and incubated for 24 hours at $37^{\circ} \mathrm{C}$. From this overnight culture, $50 \mathrm{~L}$ of bacteria was taken and spread onto solidified Muller Hinton agar, and wells were formed accordingly. The well was filled with the sample Phl-PLGA NPs $(25,50,75$, and $100 \mathrm{~g} / \mathrm{mL})$, and the standard control was Gentamycin $(10 \mathrm{~g} / \mathrm{mL})$. At $37^{\circ} \mathrm{C}$, the plates were incubated overnight. Using the nearest measurement scale, the inhibition zone diameter (IZD) was measured in millimeters.

\section{Results and Discussion}

Poly(lactic-co-glycolic acid) is one of the most successful biodegradable polymers (PLGA). Because of its appealing physico-chemical properties, PLGA has attracted a lot of interest among the various polymers formed to formulate polymeric nanoparticles. Based on this, the present study was designed to fabricate the phloretin-loaded PLGA nanoparticles. The physiochemical properties of the Phloretin-loaded PLGA NPs (Phl-PLGA NPs) were positive, indicating that some of the Phloretin molecule's amino groups were located on the NPs' surfaces. These results indicated that the phloretin had been coated successfully on PLGA NPs. The stability and cell adhesion of nanoparticles were found to be related to their surface charge properties. Ionic adsorption can cause phloretin-loaded NPs to interact with the negatively charged cell membrane, increasing cellular uptake. 


\subsection{Phloretin and PHL-PLGANPs FTIR Spectrum.}

The FTIR spectra of phloretin, PLGA, and Phl-PLGA NPs were captured and shown in Figure1. The FTIR spectra of native phloretin revealed characteristic bands due to the presence of different functional groups such as 3213.41, 2914.41, 1703.58, 1502.85, 1475.54, 1161.15, 1074.36, 979.84,792.14 and $580.74 \mathrm{~cm}^{-1}$ that could be attributed to $\mathrm{O}-\mathrm{H}$ stretching, $\mathrm{C}-\mathrm{H}$ stretching, $\mathrm{C}=\mathrm{O}$ stretching, $\mathrm{C}=\mathrm{C}$ stretching, $\mathrm{O}-\mathrm{H}$ bending, $\mathrm{C}-\mathrm{O}$ stretching, $\mathrm{C}=\mathrm{C}$. The observed peak corresponded to data from the literature $[28,29]$. The peak of $\mathrm{C}-\mathrm{O}-\mathrm{C}$ stretching in methyl groups is at 1,083.93, the peak of $\mathrm{C}-\mathrm{H}$ stretching in methyl groups is at $1450.67 \mathrm{~cm}^{-1}$, the peak of $\mathrm{C}=0$ stretching is at $1745.48 \mathrm{~cm}^{-1}$, the peak of $\mathrm{CH}$ stretching is at $2951.09,2995.45 \mathrm{~cm}^{-1}$, and the peak of $\mathrm{OH}$ stretching is around $3354.21 \mathrm{~cm}^{-1}$. The later bands in the FTIR spectrum are caused by the PLGA's in-plane and out-of-plane bending modes [30,31]. The hydroxyl group, $\mathrm{O}-\mathrm{H}$, stretching vibration is represented by the absorption band at $3404.36 \mathrm{~cm}^{1}$ in a PhlPLGA NPs. The band at $2954.95 \mathrm{~cm} 1$ is caused by the stretching vibration of alkane $\mathrm{C}-\mathrm{H}$. The carbonyl $\mathrm{C}=\mathrm{O}$ stretch FTIR spectrum peak appeared at 1747.51 and $1654.92 \mathrm{~cm}^{-1}$. The aromatic $\mathrm{C}=\mathrm{C}$ stretch and aromatic $\mathrm{C}-\mathrm{O}$ stretching are responsible for the bands at 1501.25 and $1423.47 \mathrm{~cm}^{-1}$. The spectra of Phloretin and Phl-PLGANPs have nearly identical characteristic peaks, with minor differences. This could be due to a chemical interaction between phloretin and the PLGA matrix. Furthermore, the ester linkage was used to chemically conjugate phloretin with the terminal end group of PLGA. The peak of $1273.02 \mathrm{~cm}^{-1}$ was confirmed in Phl-PLGA NPs [32]. The appearance of the IR peak was similar to other reports, and its appearance was responsible for the stabilizing and capping effects of nanoparticles $[33,34]$.

\subsection{XRD analysis of Phloretin and Phl-PLGA-NPs.}

XRD patterns of phloretin, PLGA, and Phl-PLGA NPs are shown in Figure 2. Native phloretin's sharp characteristic peaks of $12.16^{\circ}, 15.83^{\circ}, 19.66^{\circ}, 22.60^{\circ}$, and $28.87^{\circ}$ suggest its high crystalline structure. The absence of an acute peak in the PLGA molecule indicates that it is amorphous[35]. In contrast, no strong crystalline peak is observed in Phl-PLGA NPs. A less intense peak of $12.37^{\circ}, 29.67^{\circ}$, and $40.91^{\circ}$ was recorded, however. Phloretin nanoparticles were found in the PLGA matrix that was neither molecularly dispersed nor amorphous. In PLGA-based nanoparticles, others [36,37] observed a similar line of XRD patterns. Phloretin was successfully diffused into the polymeric platform to kill cancer cells thanks to the amorphous nature of the NPs.

\subsection{SEM, TEM and DLS Analysis.}

The shape, size, and surface morphology of the PLGA-NPs and Phl-PLGA NPs were examined using electronic microscopic analysis. The SEM photograph depicts the threedimensional surface topography of the Phl-PLGA NPs, which vary in size from 12.35 to 44.06 $\mathrm{nm}$. It was found to have a distinctive spherical shape (Figure 3b). The TEM micrograph reveals the particles' distinct shape, surface alteration, and polymer incorporation, and it revealed a uniform distribution of nearly spherical NPs (Figure 3d). It was discovered that the synthesized nanoparticles had a higher polydispersity index and a lower degree of uniformity in their nanosized distribution. Because of their nano-shaped and size, nanoparticles have a major effect on the combination of phloretin and cancerous cell targeting during the drug delivery process [38]. 
a)

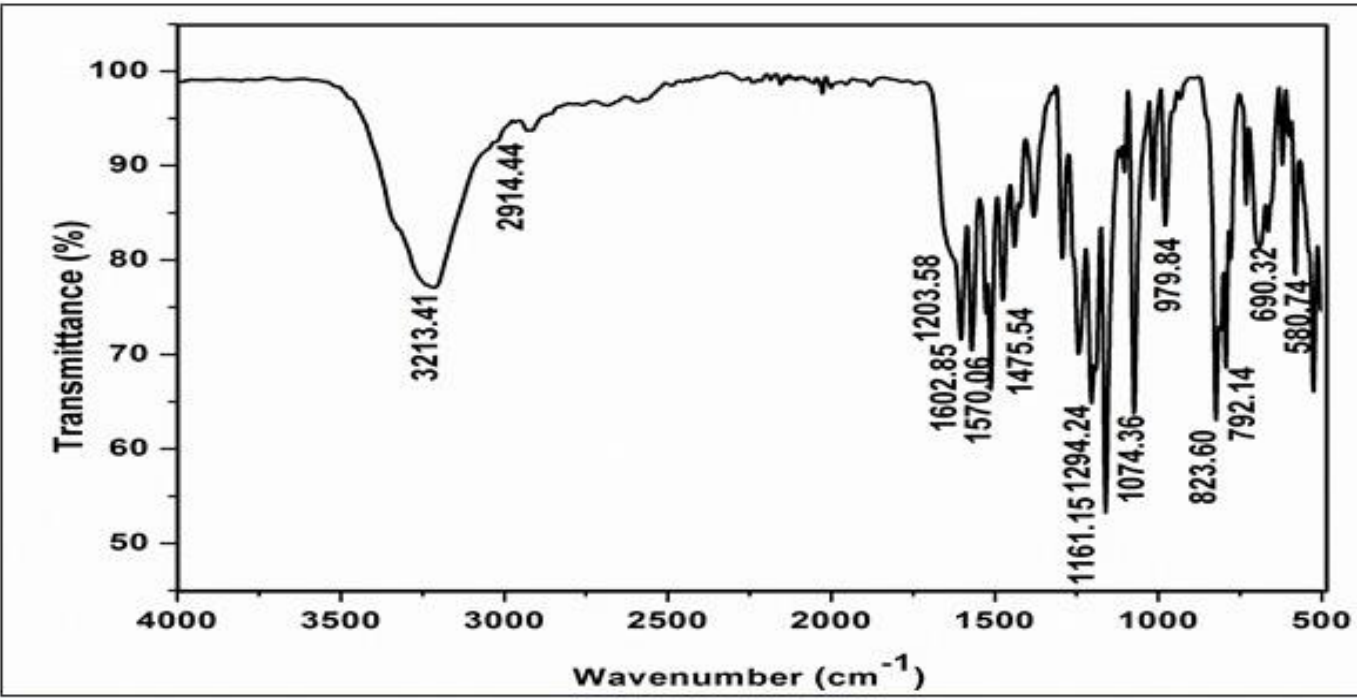

b)

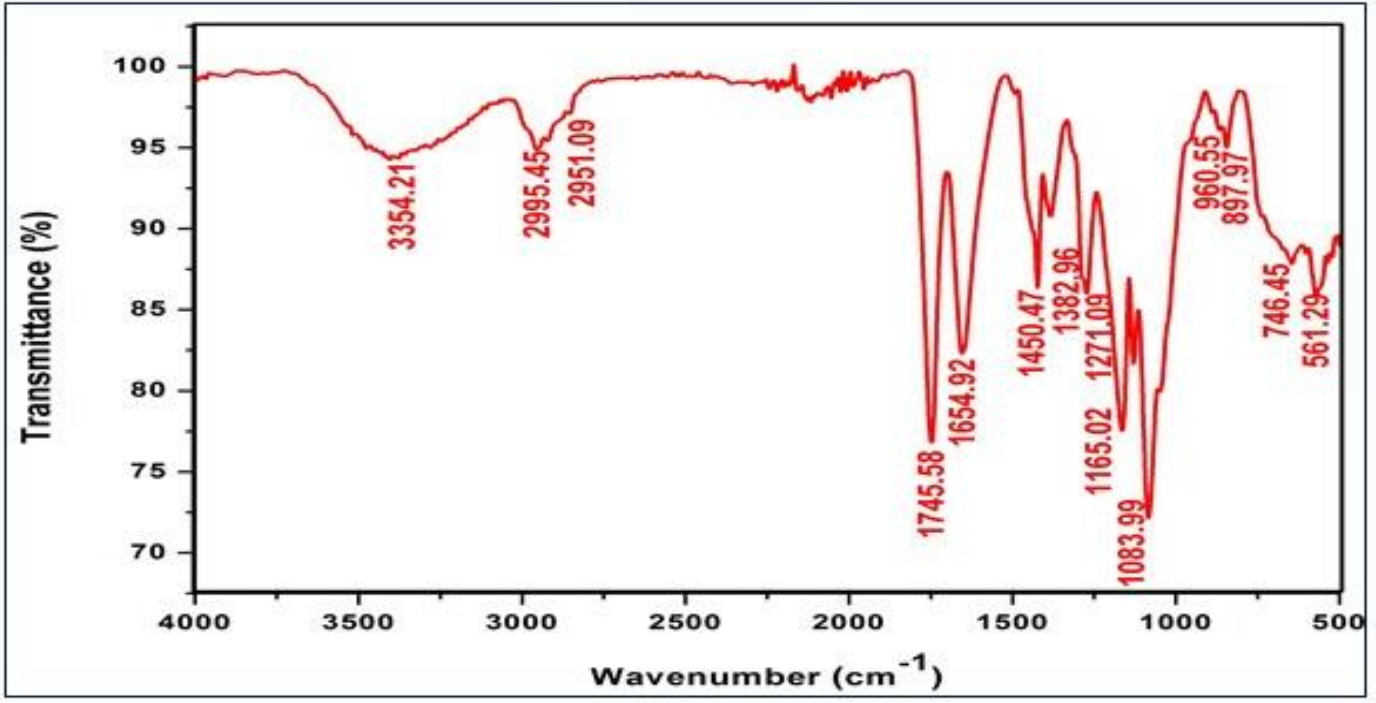

c)

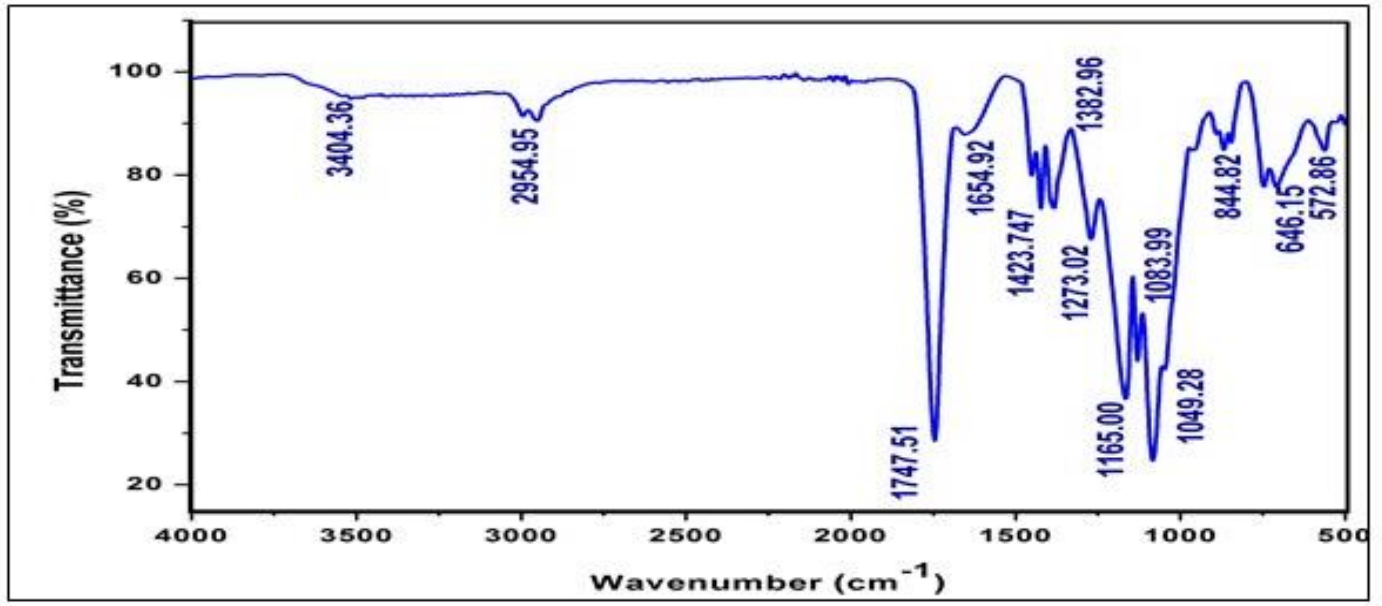

Figure 1. FTIR analysis of (a) Phloretin; (b) PLGA; (c)Phloretin loaded PLGANanoparticles (Phl-PLGA NPs). 

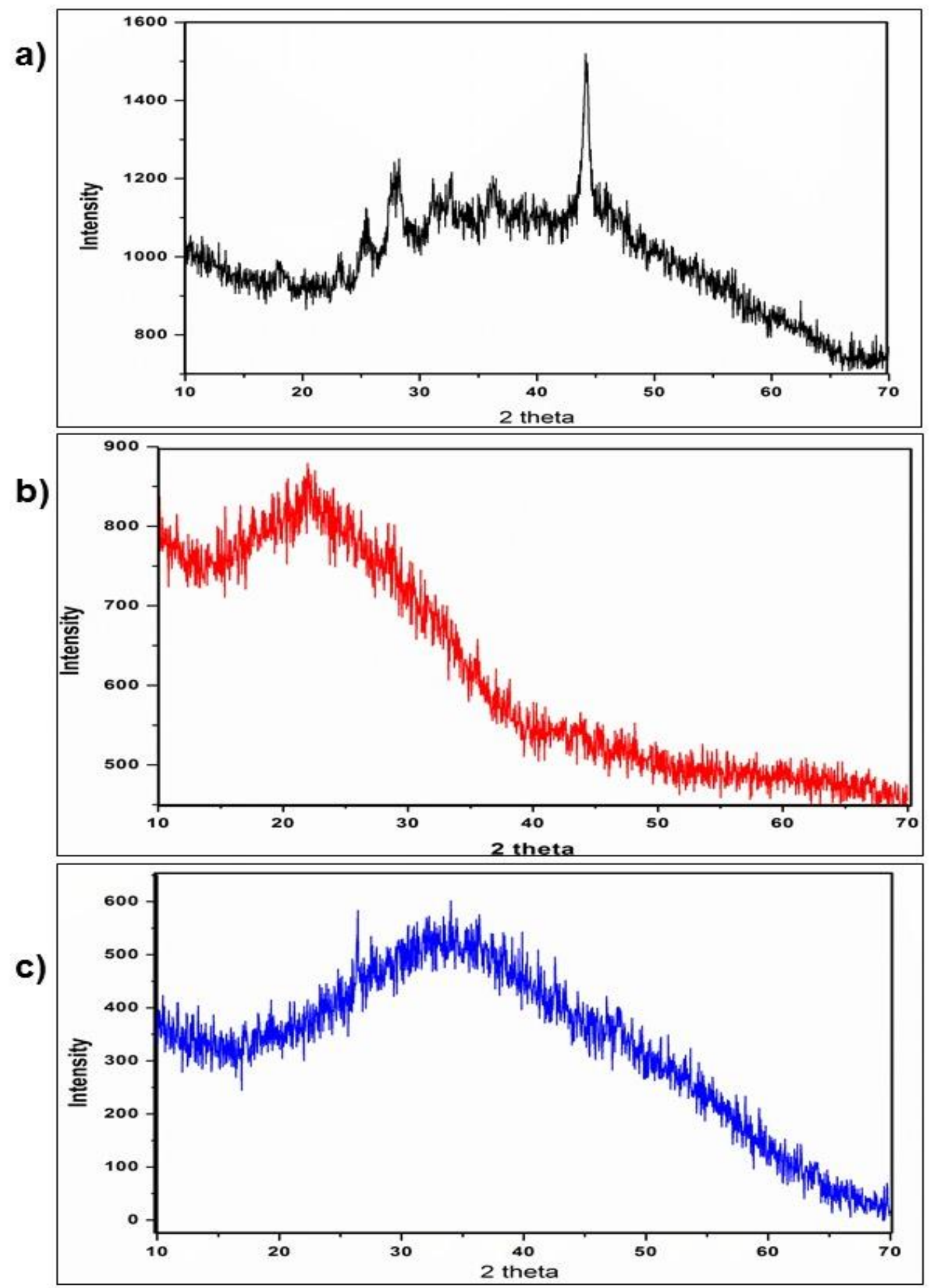

Figure 2. XRD analysis of Phloretin, PLGA, and Phloretin loadedPLGANanoparticles (Phl-PLGA NPs) 

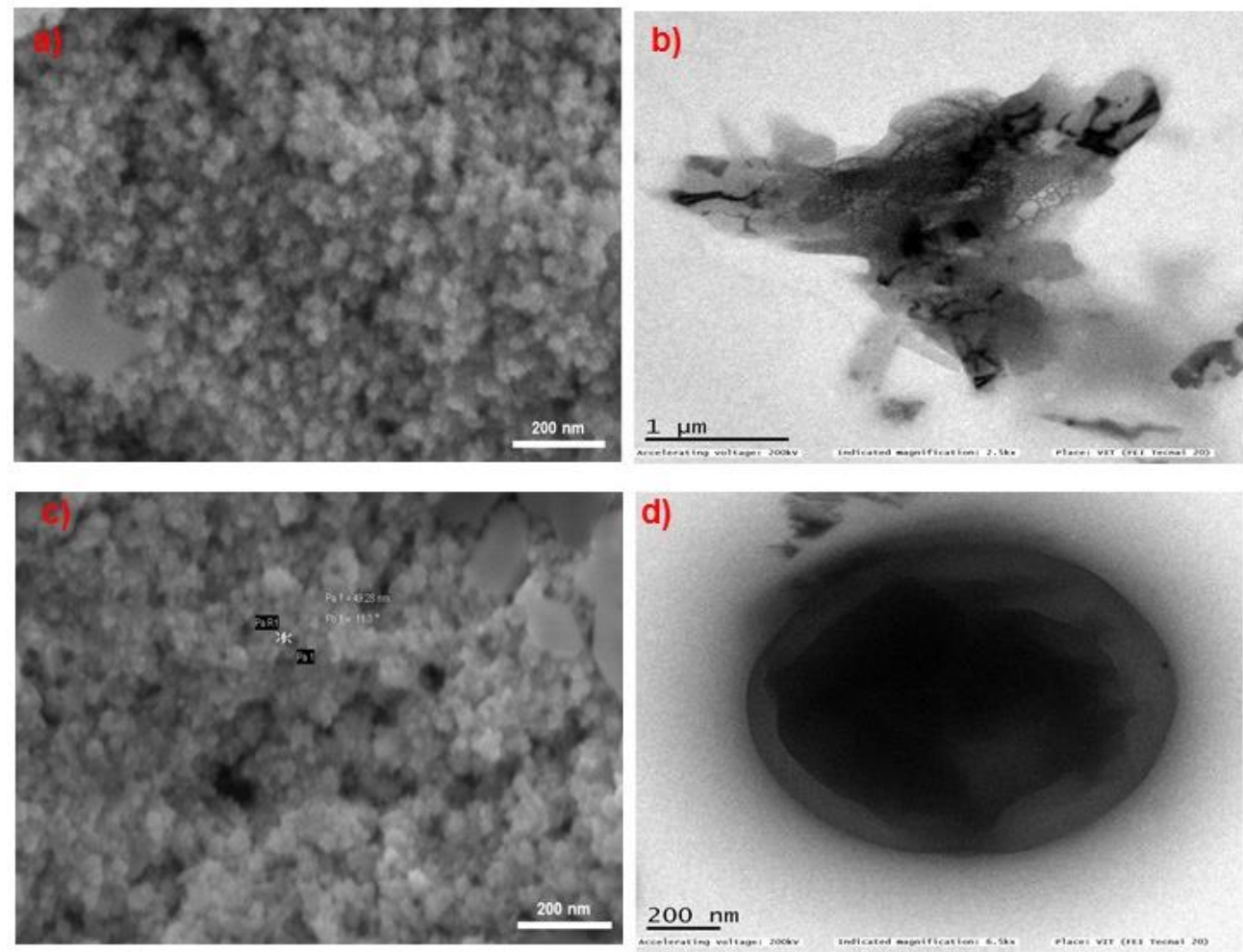

Figure 3: SEM and TEM analysis of PLGA NPs and Phloretin loadedPLGANanoparticles (Phl-PLGA NPs). (a) SEM; (b) TEM microphotograph of PLGA NPs; (c) SEM; (b) TEM microphotograph of Phl-PLGA NPs.

To evaluate the particle size, size distribution, polydispersity index, and zeta potential of nanoparticles, dynamic light scatting analysis was widely used. As shown in Figure 4, native polydispersity PLGA nanoparticles with a polydispersity index (PDI) of 0.253 and a positive zeta potential of -42.1 were discovered. Also discovered were Phl-PLGA NPs with an average diameter of $98.7 \mathrm{~nm}$, polydispersity, a polydispersity index (PDI) of 0.110 , and positive zeta potential of -45.3 . (Figure $4 \mathrm{~b}$ ). This result aligns with the previous one, which indicated that the PLGA-based nanoparticles' high positive nature makes them a promising cancer treatment candidate.
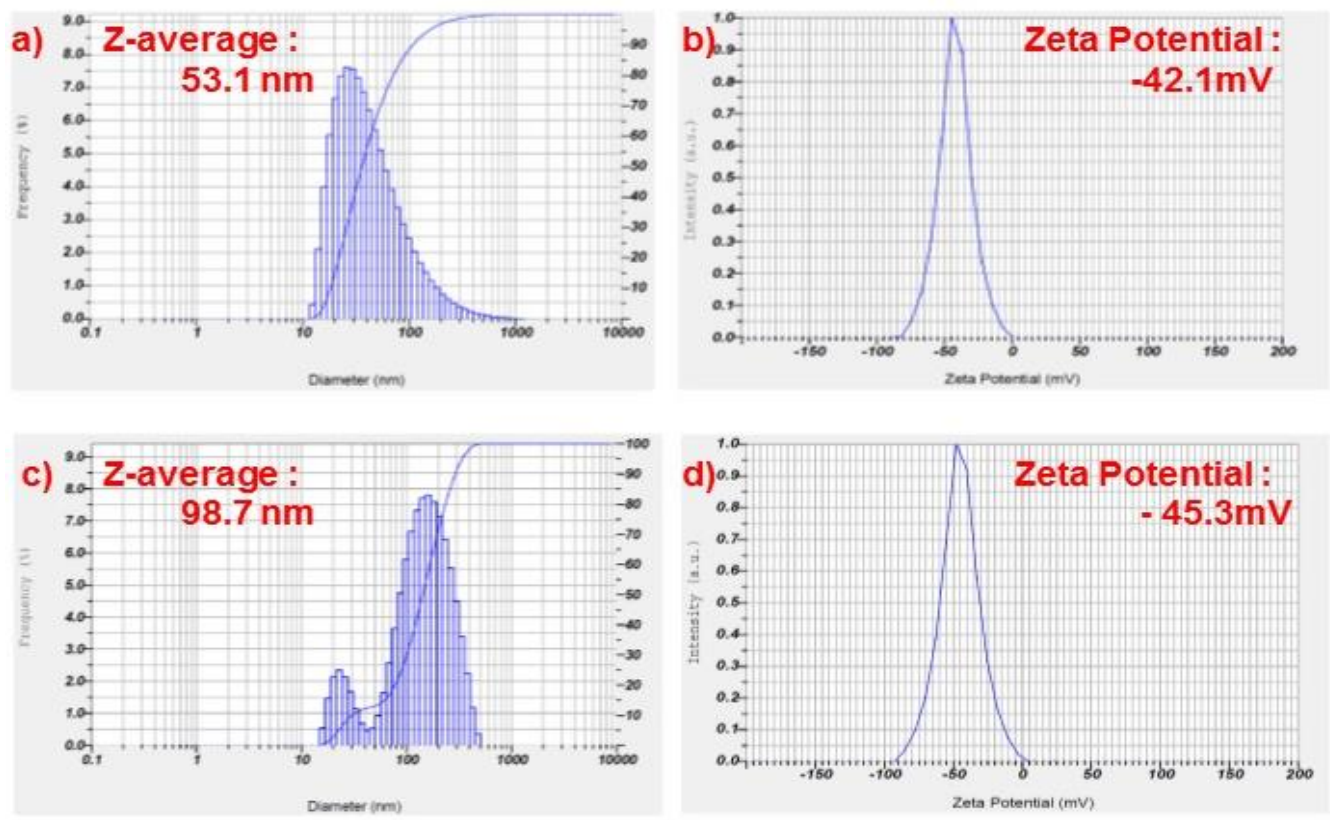

Figure 4. DLS analysis of PLGA NPs and PLGA loaded Phloretin Nanoparticles (Phl-PLGA NPs). (a) Zeta average size; (b) Zeta potential of PLGA NPs; (c) Zeta average size; (b) Zeta potential of Phl-PLGA NPs. 


\subsection{Antioxidants activity of phloretin and Phl-PLGA NPs.}

Different concentrations of Phl-PLGA NPs, ranging from 12.5 to $100 \mu \mathrm{g} / \mathrm{mL}$, were tested for antioxidant activity using various in vitro assays, including DPPH, ABTS, superoxide dismutase (SOD), Hydroxy radical $(\mathrm{OH})$, and Hydrogen peroxide $\left(\mathrm{H}_{2} \mathrm{O}_{2}\right)$ radical scavenging activity (Figure 5). In all methods, the test compound of Phl-PLGA NPs was found to scavenge free radicals in a dose-dependent manner. Phloretin, Phl-PLGA NPs ascorbic acid had a significant scavenging activity against DPPH ranging from $6.65 \pm 1.56$ to $52.23 \pm 1.80$, $10.75 \pm 1.91$ to $57.24 \pm 2.88$ and $25.41 \pm 1.57$ to $91.25 \pm 2.09 \%$, respectively.
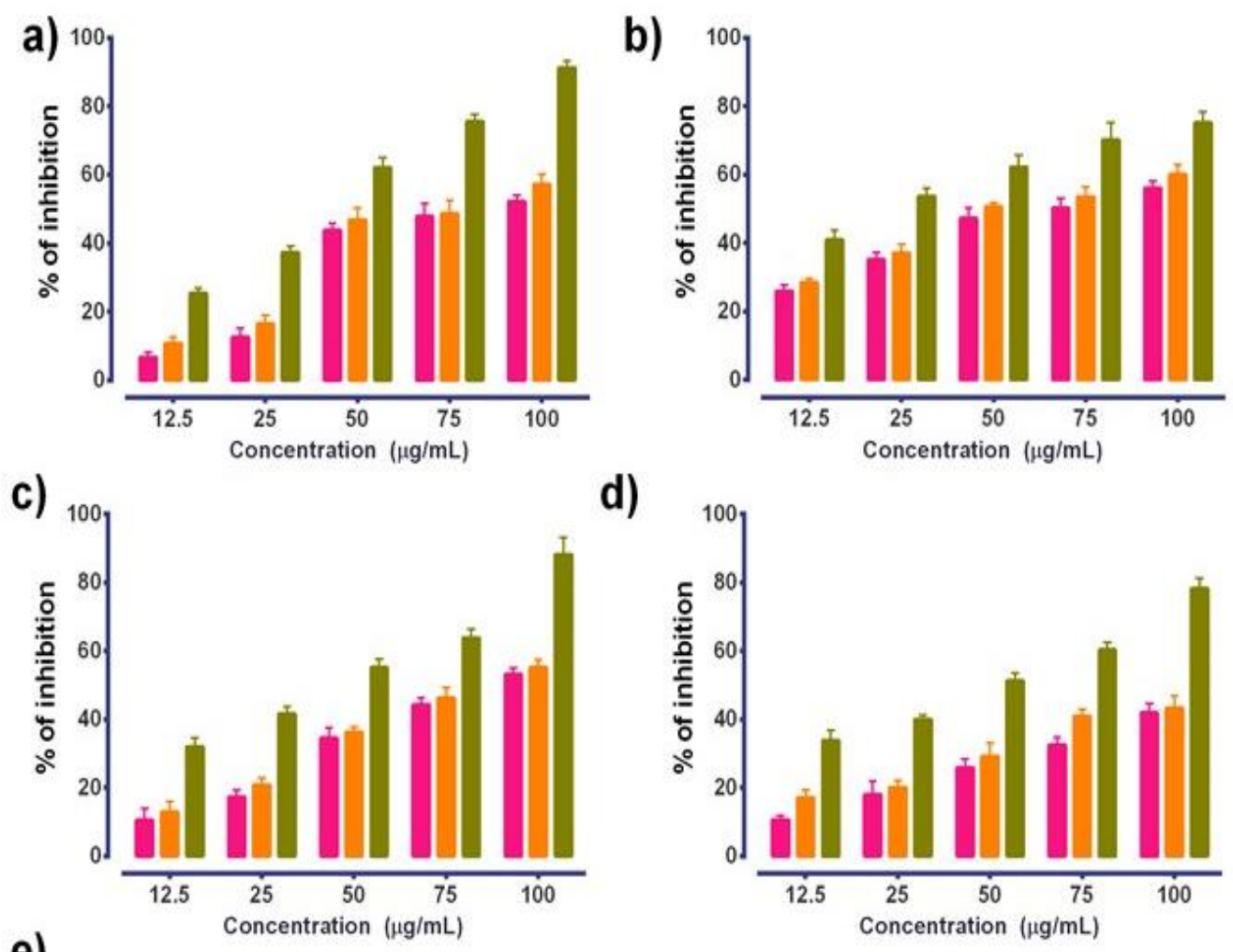

d)

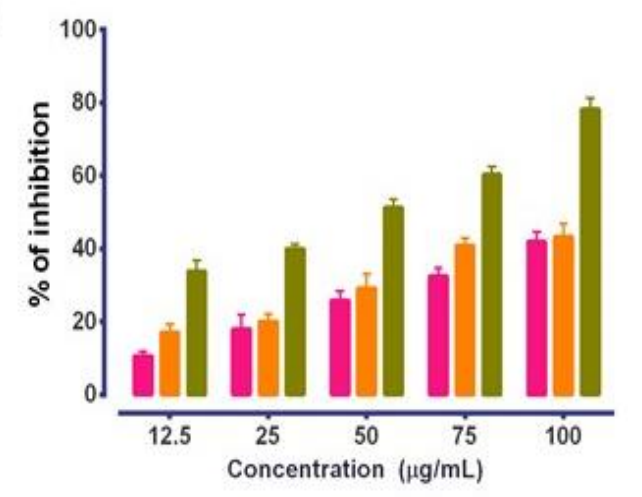

e)

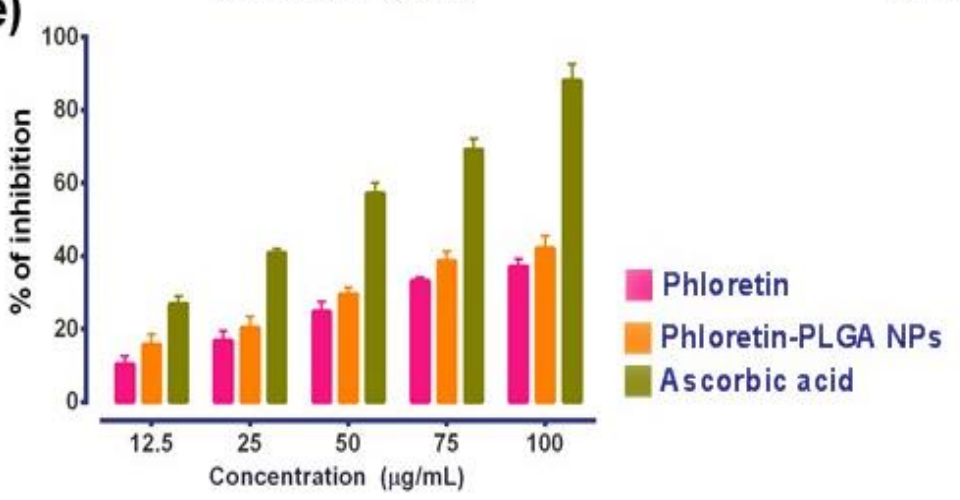

Figure 5. Antioxidants and free radical scavenging ability of bare phloretin, Phl-PLGA NPs. (a) DPPH; (b) ABTS; (c)Hydrogen peroxide; (d) Hydroxy radical and (e)superoxide dismutase radical scavenging activity of bare phloretin, Phl-PLGA NPs, and standard ascorbic acid. The value was expressed as Mean \pm SD for three independent experiments.

The ABTS free radical scavenging effect Phloretin, Phl-PLGA NPs ascorbic acid was found in the range of $25.94 \pm 1.87$ to $56.15 \pm 1.05,28.54 \pm 1.04$ to $60.19 \pm 2.72$ and $40.96 \pm 2.84$ to $75.15 \pm 3.15$, respectively. On the other hand, Hydrogen peroxide radical scavenging ability of Phloretin, Phl-PLGA NPs ascorbic acid was found in the range of $10.54 \pm 3.45$ to $53.15 \pm 1.95$, $12.98 \pm 3.05$ to $55.15 \pm 2.33$, and $32.02 \pm 2.58$ to $88.24 \pm 4.84$, respectively. Similarly, Hydroxyl radical scavenging ability of Phloretin, Phl-PLGA NPs ascorbic acid was found in the range of 
$10.65 \pm 1.28$ to $41.96 \pm 2.65,17.14 \pm 2.15$ to $43.34 \pm 3.54$, and $33.86 \pm 3.01$ to $78.24 \pm 2.64$, respectively. Superoxide scavenging activity confirmed that Phloretin, Phl-PLGA NPs ascorbic acid was found in the range of $10.47 \pm 2.08$ to $37.24 \pm 2.03,15.83 \pm 2.71$ to $42.14 \pm 3.33$ and $26.97 \pm 2.05$ to $88.12 \pm 4.03$, respectively. It shows that the fabricated Phl-PLGA NPs have a significant antioxidant activity when compared with standard ascorbic acid. It shows that the fabricated Phl-PLGA NPs have more antioxidants by observing the results of DPPH, ABTS, SOD, $\mathrm{OH}$, and $\mathrm{H}_{2} \mathrm{O}_{2}$ radical scavenging assay, it clearly shows that the fabricated Phl-PLGA NPs have high antioxidant potential; thus, it quenched the stable free radicals in the reaction Hence, to improve the bioavailability of Phloretin, Phl-PLGA NPs would contribute more antioxidant profile in the conditions of oxidative damages.

Table 1. Antibacterial activity of Phl-PLGA NPs against gram-negative pathogenic bacteria.

\begin{tabular}{l|l|l|l|l|l}
\multirow{2}{*}{ Microorganism } & \multicolumn{4}{|c|}{ Phl-PLGA NPs } & Tetracycline \\
\cline { 2 - 6 } & $\mathbf{2 5} \boldsymbol{\mu \mathbf { g } / \mathbf { m L }}$ & $\mathbf{5 0} \boldsymbol{\mu \mathbf { g } / \mathbf { m L }}$ & $\mathbf{7 5} \boldsymbol{\mu \mathbf { g } / \mathbf { m L }}$ & $\mathbf{1 0 0} \boldsymbol{\mu \mathbf { g } / \mathbf { m L }}$ & $\mathbf{1 0} \boldsymbol{\mu \mathbf { g } / \mathbf { m L }}$ \\
\hline E.coli & $10.8 \pm 0.91$ & $13.4 \pm 0.32$ & $16.1 \pm 0.40$ & $23.8 \pm 0.50$ & $25.11 \pm 1.06$ \\
\hline P.aeruginosa & $10.3 \pm 0.35$ & $11.6 \pm 0.32$ & $16.6 \pm 0.10$ & $23.6 \pm 0.10$ & $27.42 \pm 0.25$ \\
Value is expressed for three independent experiments.
\end{tabular}

\subsection{Antimicrobial activity.}

This was planned to use synthesized Phl-PLGA NPs as a substitute for conventional antibiotics which fight against intracellular pathogenic bacteria, particularly drug-resistant bacterial strains including Escherichia coli and Pseudomonas aeruginosa. The antimicrobial activity of Phl-PLGA NPs against bacterial strains. The antimicrobial actions of the Phl-PLGA NPs were increased with an increase in the concentration of Phl-PLGA NPs compared with the standard drug. The antimicrobial sensitivity results showed that the highest inhibition zone was measured in E.coli $(23.8 \pm 0.50 \mathrm{~nm})$ and P.aeruginosa $(23.6 \pm 0.10 \mathrm{~nm})$ at $100 \mu \mathrm{g} / \mathrm{mL}$, which indicates that Phl-PLGA NPs has a broad-spectrum antibiotic potential to act against gramnegative bacteria. Mary et al. (2018) confirmed that AgNPs had antibacterial activity against Gram-negative bacteria in a previous study[40]. Although the mechanism of action of polymer bassed nanoparticles against negative bacteria is unknown, the most widely accepted theories are that the interaction of nanoparticles might be increases permeability of the bacterial cell membrane, allowing intracellular content to be disrupted and extravasated and that the development of reactive oxygen species (ROS) as a result of the interaction with nanoparticle inhibits the bacterial growth.

\section{Conclusions}

Attending this investigation reveals an effect of Phl-PLGA NPs on antioxidant and antimicrobial profiles. The results showed the evident benefits of Phl-PLGA NPs, which could be taken for various pathophysiological conditions upon bacterial diseases and oxidative stressrelated conditions. The location of this fabricated bio polymer-based phytonanotherapy has a great pipeline in finding medications against antibacterial resistant factors and antioxidant defenses. The synthesis of PLGA-formulated Phloretin-loaded nanoparticles is revealed in this study. The synthesized nanoparticles are mainly sphere formed, with an average size of 12.35 to $44.06 \mathrm{~nm}$, according to the results of DLS, SEM, and TEM studies. The crystalline nature and molecular composition of the synthesized nanoparticles were verified by FTIR and XRD 
results. According to the study's findings, phloretin-loaded PLGA nanoparticles may be used as a good source of antioxidants and antibacterial agents. However, more research is required in this field to promote this formulation as a nanomedicine therapeutic molecule.

\section{Funding}

This research received no external funding.

\section{Acknowledgments}

The authors are thankful to the authorities of Sri Akilandeswari Women's College, Wandiwash, Tamil Nadu, India, for providing the necessary support to carry out this research work.

\section{Conflicts of Interest}

The authors declare no conflict of interest.

\section{References}

1. Alimardani, V.; Abolmaali, S.S.; Tamaddon, A.M. Recent advances on nanotechnology-based strategies for prevention, diagnosis, and treatment of coronavirus infections. Journal of Nanomaterials 2021, 2021, 9495126, https://doi.org/10.1155/2021/9495126.

2. Lagreca, E.; Onesto, V.; Di Natale, C.; La Manna, S.; Netti, PA.; Vecchione, R. Recent advances in the formulation of PLGA microparticles for controlled drug delivery. Progress in Biomaterials 2020, 9, 1-22, https://doi.org/10.1007/s40204-020-00139-y.

3. Makadia, H.K, Siegel, S.J. Poly lactic-co-glycolic acid (PLGA) as biodegradable controlled drug delivery carrier. Polymers 2011, 3, 1377-97, https://doi.org/10.3390/polym3031377.

4. Su, S.; Kang, P.M. Systemic review of biodegradable nanomaterials in nanomedicine. Nanomaterials. 2020, 10,656, https://doi.org/10.3390/nano10040656.

5. Derman, S. Caffeic acid phenethyl ester loaded PLGA nanoparticles: effect of various process parameters on reaction yield, encapsulation efficiency, and particle size. Journal of Nanomaterials 2015, 1, 2015, https://doi.org/10.1155/2015/341848.

6. Yoo, J.; Won, Y.Y. Phenomenology of the initial burst release of drugs from PLGA Microparticles. ACS Biomaterials Science \& Engineering 2020, 6, 6053-6062, https://doi.org/10.1021/acsbiomaterials.0c01228.

7. De Jalón.; E.G, Blanco-Prıeto, M.J.; Ygartua, P.; Santoyo, S. PLGA microparticles: possible vehicles for topical drug delivery. International Journal of Pharmaceutics 2001, 226, 181-184, https://doi.org/10.1016/S0378-5173(01)00811-0.

8. Zhang, X.; Sun, M.; Zheng, A.; Cao, D.; Bi, Y.; Sun, J. Preparation and characterization of insulin-loaded bioadhesive PLGA nanoparticles for oral administration. European Journal of Pharmaceutical Sciences 2012,45,632-8, https://doi.org/10.1016/j.ejps.2012.01.002.

9. Mariadoss, A.V.; Vinyagam, R.; Rajamanickam,V.; Sankaran,V.;Venkatesan, S.; David, E. Pharmacological aspects and potential use of phloretin: A systemic review. Mini Reviews in Medicinal Chemistry 2019,19,1060-7, https://doi.org/10.2174/1389557519666190311154425.

10. Tsao, R.; Yang, R.; Young, J.C.; Zhu, H. Polyphenolic profiles in eight apple cultivars using highperformance liquid chromatography (HPLC). Journal of Agricultural and Food Chemistry 2003, 51, 634753, https://doi.org/10.1021/jf0346298.

11. Ali, S.S.; Ahsan, H.; Zia, M.K.; Siddiqui, T.; Khan, F.H. Understanding oxidants and antioxidants: classical team with new players. Journal of Food Biochemistry 2020, 44, https://doi.org/10.1111/jfbc.13145.

12. Wang, Y.; Li, D.; Lin, H.; Jiang, S.; Han, L.; Hou, S.; Lin, S.; Cheng, Z.; Bian, W.; Zhang, X.; He, Y. Enhanced oral bioavailability and bioefficacy of phloretin using mixed polymeric modified selfnanoemulsions. Food Science \& Nutrition 2020, 8, 3545, https://doi.org/10.1002/fsn3.1637.

13. Abu-Azzam. O.; Nasr, M. In vitro anti-inflammatory potential of phloretin microemulsion as a new formulation for prospective treatment of vaginitis. Pharmaceutical Development and Technology 2020, 25, 930-5, https://doi.org/10.1080/10837450.2020.1764032. 
14. Kalinowska, M.; Gryko, K.; Wróblewska, A.M.; Jabłońska-Trypuć, A.; Karpowicz, D. Phenolic content, chemical composition and anti-/pro-oxidant activity of Gold Milenium and Papierowka apple peel extracts. Scientific Reports 2020, 10, 1-5, https://doi.org/10.1038/s41598-020-71351-w.

15. Mariadoss, A.V.; Vinayagam, R.; Xu, B.; Venkatachalam, K.; Sankaran, V.; Vijayakumar, S.; Bakthavatsalam, S.R.; Mohamed, S.A.; David, E. Phloretin loaded chitosan nanoparticles enhance the antioxidants and apoptotic mechanisms in DMBA induced experimental carcinogenesis. ChemicoBiological Interactions 2019, 308, 11-9, https://doi.org/10.1016/j.cbi.2019.05.008.

16. Mariadoss, A.V.; Vinayagam, R.; Senthilkumar, V.; Paulpandi, M.; Murugan, K.; Xu, B.; Gothandam, K.M.; Kotakadi,V.S.; David, E. Phloretin loaded chitosan nanoparticles augments the pH-dependent mitochondrial-mediated intrinsic apoptosis in human oral cancer cells. International Journal of Biological Macromolecules 2019, 130, 997-1008, https://doi.org/10.1016/j.ijbiomac.2019.03.031.

17. Mariadoss, A.V.A.; Dhanabalan, A.K.; Munusamy, H.; David, E. In silico studies towards enhancing the anti-cancer activity of phytochemical phloretin against cancer drug targets. Current Drug Theraphy 2018, 13, 174-88, https://doi.org/10.2174/1574885513666180402134054.

18. Gosch, C.; Halbwirth, H.; Stich, K. Phloridzin: biosynthesis, distribution and physiological relevance in plants. Phytochemistry 2010, 71, 838-843, https://doi.org/10.1016/j.phytochem.2010.03.003.

19. Vinayagam, R.; Lee, K.E.; David, E.; Matin, M.N.; Kang, S.G. Facile green preparation of PLGA nanoparticles using wedelolactone: its cytotoxicity and antimicrobial activities. Inorganic Chemistry Communications 2021,108583, https://doi.org/10.1016/j.inoche.2021.108583.

20. Krithiga, B.; Casimeer, S.C.; Ghidan, A.Y.; Ghethan F.Y.; Venkatachalam, K.; Singaravelu, A. Bioformulated hesperidin-loaded PLGA nanoparticles counteract the mitochondrial-mediated intrinsic apoptotic pathway in cancer cells. Journal of Inorganic and Organometallic Polymers and Materials 2020, 31, 331-343, https://doi.org/10.1007/s10904-020-01746-9.

21. Bedlovicová, Z.; Strapá, I.; Baláž, M.; Salayová, A. A Brief Overview on Antioxidant Activity Determination of Silver Nanoparticles. Molecules 2020, 25, 3191, https://doi.org/10.3390/molecules25143191.

22. Braca, A.; Tommasi, N.D.; Bari, L.D.; Pizza, C.; Politi, M.; Morell, I.I. Antioxidant principles from Bauhinia tetrapotensis. Journal of Natural Products 2001, 64, 892-895, https://doi.org/10.1021/np0100845.

23. Re. R.; Pellegrini, N.; Proteggente, A.; Pannala, A.; Yang, M.; Rice-Evans, C. Antioxidant activity applying an improved ABTS radical cation decolorization assay. Free Radical Biology and Medicine 1999, 26,12317, https://doi.org/10.1016/S0891-5849(98)00315-3.

24. Arai, Y.; Park, H.; Park, S.; Kim, D.; Baek, I.; Jeong, L.; Kim.; B.J.; Park, K.; Lee, D.; Lee, S.H. Bile acidbased dual-functional prodrug nanoparticles for bone regeneration through hydrogen peroxide scavenging and osteogenic differentiation of mesenchymal stem cells. Journal of Controlled Release 2020, 328, 596607, https://doi.org/10.1016/j.jconrel.2020.09.023.

25. Kumar, S.P.; Birundha, K.; Kaveri, K.; Devi, K.R. Antioxidant studies of chitosan nanoparticles containing naringenin and their cytotoxicity effects in lung cancer cells. International Journal of Biological Macromolecule 2015, 78, 87-95, https://doi.org/10.1016/j.ijbiomac.2015.03.045.

26. Pool, H.; Quintanar, D.; Figueroa, J.;D.; Marinho Mano, C.; Bechara, J.;E;; Godínez, L.;A,; Mendoza, S. Antioxidant effects of quercetin and catechin encapsulated into PLGA nanoparticles. Journal of Nanomaterials 2012, 2012, https://doi.org/10.1155/2012/145380.

27. Barreca, D.; Bellocco, E.; Laganà, G.; Ginestra, G.; Bisignano, C. Biochemical and antimicrobial activity of phloretin and its glycosilated derivatives present in apple and kumquat. Food Chemistry 2014, 160, 292-7, https://doi.org/10.1016/j.foodchem.2014.03.118.

28. Gao, Y.; Anand, M.A.; Ramachandran, V.; Karthikkumar, V.; Shalini, V.; Vijayalakshmi, S.; Ernest, D. Biofabrication of zinc oxide nanoparticles from Aspergillus niger, their antioxidant, antimicrobial and anticancer activity. Journal of Cluster Science 2019, 30, 937-46, https://doi.org/10.1007/s10876-019-01551-6.

29. Sulaiman, G.M.; Waheeb, H.M.; Jabir, M.S.; Khazaal, S.H.; Dewir, Y.H.; Naidoo, Y. Hesperidin loaded on gold nanoparticles as a drug delivery system for a successful biocompatible, anti-cancer, anti-inflammatory and phagocytosis inducer model. Scientific Reports 2020, 10, 1-6, https://doi.org/10.1038/s41598-02066419-6.

30. Singh, R.; Kesharwani, P.; Mehra, N.K, Singh, S, Banerjee, S.; Jain, N.K. Development and characterization of folate anchored Saquinavir entrapped PLGA nanoparticles for anti-tumor activity. Drug Development and Industrial Pharmacy 2015, 41, 1888-1901, https://doi.org/10.3109/03639045.2015.1019355. 
31. Mahassni, S.H.; Al-Reemi, R.M. Apoptosis and necrosis of human breast cancer cells by an aqueous extract of garden cress (Lepidium sativum) seeds. Saudi Journal of Biological Sciences 2013, 20, 131-9, https://doi.org/10.1016/j.sjbs.2012.12.002.

32. Vrignaud, S.; Benoit, J.P.; Saulnier, P. Strategies for the nanoencapsulation of hydrophilic molecules in $\begin{array}{lllll}\text { polymer-based } \quad \text { nanoparticles. } & \text { Biomaterials } & \text { 2011, } & 32, & \text { 8593-8604, }\end{array}$ https://doi.org/10.1016/j.biomaterials.2011.07.057.

33. Sulaiman, G.M, Waheeb, H.M, Jabir, M.S, Khazaal, S.H, Dewir, Y.H, Naidoo, Y. Hesperidin loaded on gold nanoparticles as a drug delivery system for a successful biocompatible, anti-cancer, anti-inflammatory and phagocytosis inducer model. Scientific Reports 2020, 10,1-6, https://doi.org/10.1038/s41598-020-66419-6.

34. Ali, S.H.; Sulaiman, G.M.; Al-Halbosiy, M.M.; Jabir, M.S.; Hameed, A.H. Fabrication of hesperidin nanoparticles loaded by poly lactic co-Glycolic acid for improved therapeutic efficiency and cytotoxicity. Artificial cells, Nanomedicine, and Biotechnology 2019, 47, 378-394, https://doi.org/10.1080/21691401.2018.1559175.

35. Klöpffer,W. ESCA in Polymer Spectroscopy. In Introduction to Polymer Spectroscopy 1984, Springer, Berlin, Heidelberg, https://doi.org/10.1007/978-3-642-69373-1.

36. Jain, R.A. The manufacturing techniques of various drug loaded biodegradable poly (lactide-coglycolide)(PLGA) devices. Biomaterials 2000, 21, 2475-90, https://doi.org/10.1016/s0142-9612(00)001150 .

37. Majumdar, S.; Srirangam, R. Solubility, stability, physicochemical characteristics and in vitro ocular tissue permeability of hesperidin: a natural bioflavonoid. Pharmaceutical Research 2009, 26, 1217-25, https://doi.org/10.1007/s11095-008-9729-6.

38. Cho, S.A.; Cha, S.R.; Park, S.M.; Kim, K.H.; Lee, H.G.; Kim, E.Y.; Lee, D.; Khang, G. Effects of hesperidin loaded poly (lactic-co-glycolic acid) scaffolds on growth behavior of costal cartilage cells in vitro and in vivo. Journal of Biomaterials Science 2014, 25, 625-640, https://doi.org/10.1080/09205063.2014.888304.

39. Dang, Y.; Guan, J. Nanoparticle-based drug delivery systems for cancer therapy. Smart Materials in Medicine 2020, 1, 10-19, https://doi.org/10.1016/j.smaim.2020.04.001.

40. Mikhailova, E.O. Silver nanoparticles: mechanism of action and probable bio-application. Journal of Functional Biomaterials 2020, 11, https://doi.org/10.3390/jfb11040084. 